\title{
Formulation of and adherence to a care plan for potentially frail community-dwelling older people by practice nurses in the Netherlands
}

\author{
Mandy M. N. Stijnen ${ }^{1}$, Hubertus J . M. Vrijhoef ${ }^{2,3}$, Inge G. P. Duimel-Peeters ${ }^{1,4}$ Maria W. J . \\ J ansen ${ }^{5,6}$
}

1. Department of Family Medicine, School for Public Health and Primary Care (CAPHRI), Maastricht University, Maastricht, The Netherlands. 2. Tilburg School of Social and Behavioral Sciences, Scientific Centre for Care and Welfare (TRANZO), Tilburg University, Tilburg, The Netherlands. 3. School for Public Health, National University of Singapore, Singapore. 4. Department of Patient \& Care, Maastricht University Medical Centre, Maastricht, The Netherlands. 5. Academic Collaborative Centre for Public Health Limburg, Public Health Service Southern Limburg, Geleen, The Netherlands. 6. School for Public Health and Primary Care (CAPHRI), Maastricht University, Maastricht, The Netherlands.

Correspondence: Mandy M. N. Stijnen. Address: Department of Family Medicine, School for Public Health and Primary Care (CAPHRI), Maastricht University, Maastricht, The Netherlands. Email: mandy.stijnen@maastrichtuniversity.nl

Received: November 24, 2013

DOI : $10.5430 /$ jnep.v4n8p34
Accepted: January 6, 2014

URL: http://dx.doi.org/10.5430/jnep.v4n8p34

\section{Abstract}

Background: In the Netherlands, practice nurses within general practices increasingly deliver proactive care to older people. As part of a home visitation programme, they assess older people's health status during a home visit, followed by formulating a care plan and monitoring follow-up care. Insight into the latter two steps is limited, whereas previous research shows signs of inadequate follow-up, threatening the achievement of optimal patient outcomes. The objectives of the current study are to investigate practice nurses' actions and views regarding (1) the formulation of a care plan for potentially frail community-dwelling older people following a comprehensive geriatric assessment during a home visit and (2) their adherence to the care plan and monitoring during follow-up of older people in whom health and/or well-being problems have been detected.

Methods: A cross-sectional mixed-model study was conducted. Results of the comprehensive geriatric assessment were compared to the care plans to reveal the percentage of problems included. Semi-structured interviews were performed with 12 practice nurses who visited older people at home. Per practice nurse, care plans of 10 older people (total $n=120$ ) were purposefully selected and discussed to identify reasons for not registering problems in the care plan and to determine whether and how problems were monitored.

Results: Of the problems identified through comprehensive geriatric assessment, 27.8\% $(n=275)$ were included in a care plan of which $62.9 \%(n=173)$ were addressed using follow-up actions. Patient-related and problem-specific factors influenced the formulation of care plans. Insufficient time challenged practice nurses in monitoring older people over time.

Conclusions: The formulation of care plans and monitoring older people over time offers room for improvement. Once problems are included into the plans, practice nurses mostly adhere to the care plans. 


\section{Key words}

Frail elderly, General practice, Geriatric assessment, Patient care planning, Practice nurse

\section{I ntroduction}

As the ageing of the population continues, the accompanying rise in frail older people poses a high burden on the healthcare system. Frail older people are at increased risk of adverse outcomes such as falls, disability, and hospitalisation ${ }^{[1]}$. In targeting frail older people, early detection of problems and needs seems beneficial as it offers primary care professionals an opportunity to discuss goals of care and informs proactive decisions in planning care, treatment, and well-being services ${ }^{[2]}$. As a result, within general practices in the Netherlands, shifting of care from general practitioners (GPs) towards practice nurses (PNs) ${ }^{[3]}$ has extended from chronic disease management to proactive care for potentially frail older people.

One way to organise nurse-based primary care for older people is by applying certain generic, evidence-based principles for the effective management of chronic diseases in primary care that are associated with improved health outcomes, such as targeted assessment, a personalised care plan for goal achievement, and active follow-up ${ }^{[4]}$. These elements are incorporated in a nurse-delivered preventive home visitation programme implemented in general practices in the south of the Netherlands. The [G]OLD home visitation programme ('Getting OLD the healthy way') is aimed at the early identification of health and/or well-being problems followed by, if required, individualised care and follow-up with the ultimate goal to maintain independent living among potentially frail community-dwelling older people $(\geq 75 \text { years })^{[5]}$. The PN performs an assessment of the person's health problems and needs using the [G]OLD comprehensive geriatric assessment (CGA) during a home visit. Based on the results and discussion with the GP, the PN formulates a personalised care plan with written agreements between the GP/PN and the older person concerning follow-up actions to be undertaken. Hereafter, the PN coordinates referral to care and/or well-being facilities, monitors progress, and evaluates whether changes according to the agreements written down in the care plan have occurred or new problems have arisen since the last visit.

Home visitation programmes that include extended follow-up and evaluation of the implementation of recommendations for problems identified through CGA are more likely to be effective ${ }^{[6,7]}$, although many studies do not provide details on these aspects ${ }^{[8]}$. The care providers' perspective of planning these care and treatment recommendations and organising follow-up is largely neglected. Yet, less-than-optimal patient outcomes can not only be attributed to a lack of adherence by older people to care plans but also to inadequate follow-up by the care provider ${ }^{[9]}$. A recent process evaluation of a home visitation programme found that the extent of evaluation of care and treatment recommendations and follow-up over time by care providers was limited ${ }^{[10]}$. Thus, the aim of this study was to investigate PNs' actions and views regarding (1) the formulation of a care plan for potentially frail community-dwelling older people following a CGA during a home visit and (2) their adherence to the care plan and monitoring during follow-up of older people in whom health and/or well-being problems have been detected.

\section{Methods}

\subsection{Study design}

A cross-sectional study was conducted. To yield an enriched understanding of the topic of interest, both quantitative and qualitative methods were used. This resulted in a mixed-model design, or more specifically a convergent-parallel approach and data transformation design ${ }^{[1]}$. Quantitative and qualitative data were given equal priority, they were gathered concurrently, and mixing took place at the stages of research question formulation, data analysis, and interpretation (see Figure 1). 


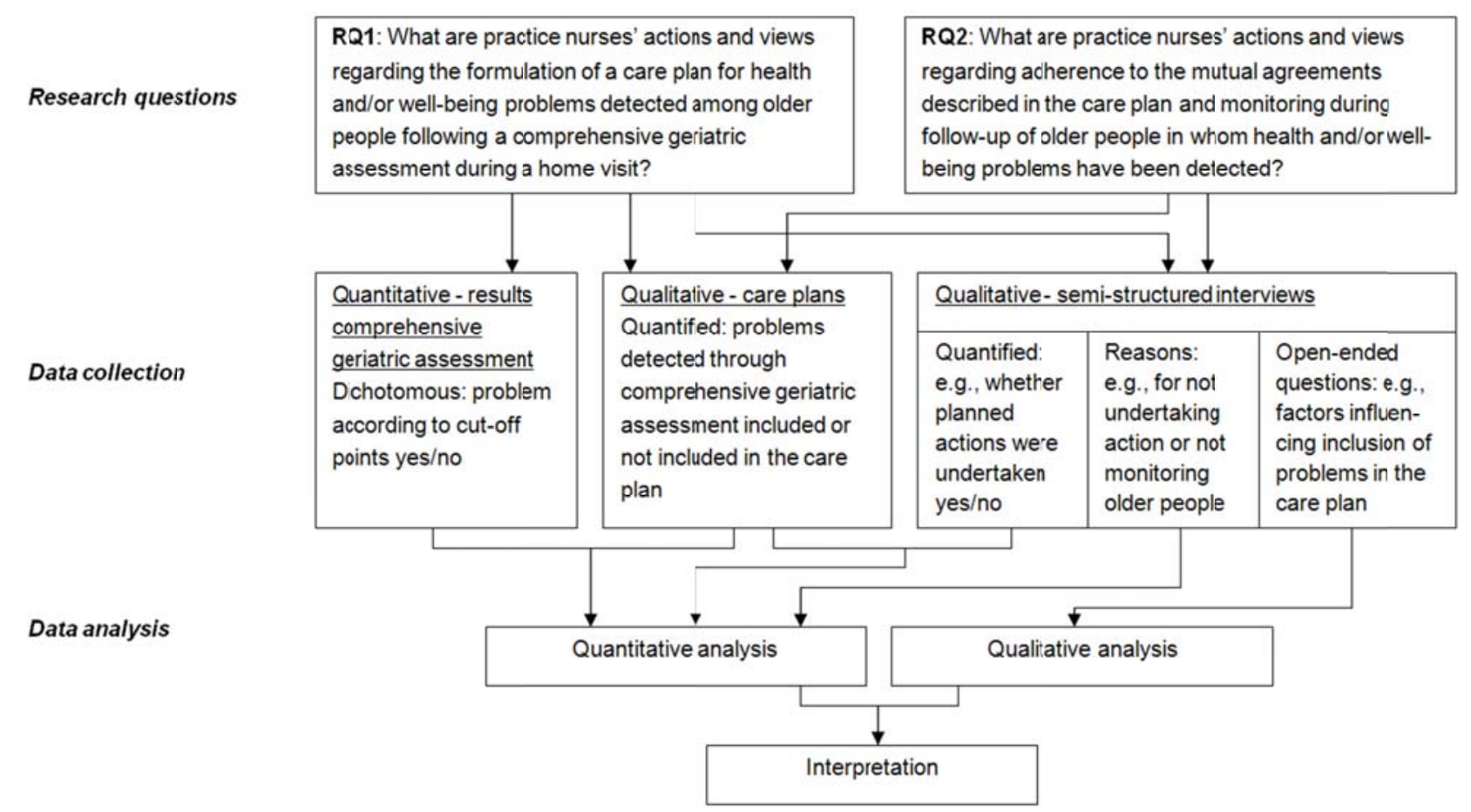

Figure 1. Visualisation of the mixed-model research design used in this study

\subsection{Setting and participants}

Thirteen PNs performed home visits on behalf of a quasi-experimental controlled trial. At that time, PNs were not informed about an interview to evaluate the formulation of and adherence to the care plans. Approximately 1 year after finalising the home visits for the trial, PNs were approached to participate in a semi-structured interview for this study. Twelve PNs (92.3\%) from 12 general practices in the south of the Netherlands verbally consented to participate; 1 PN withdrew from participation due to time constraints and work commitments.

Between July 2010 and September 2011, 12 PNs visited 565 older people, with an average of 47.1 home visits per PN (SD =19.6; range: 13-78). Per PN, we purposefully selected 10 cases they visited before in which the most health and/or well-being problems had been detected to have sufficient input for the semi-structured interviews (total $n=120$ cases). In this way, we intended to obtain a comprehensive understanding of the decision-making process, while keeping the time investment for PNs to discuss the cases to a minimum (i.e., maximum interview duration of one hour).

\subsection{Data collection}

\subsubsection{Comprehensive geriatric assessment}

Our CGA comprises an assessment by the PN of older people's physical functioning (e.g., blood pressure, mobility), psychological and mental functioning (e.g., cognition, depression), social functioning (e.g., loneliness), lifestyle, and medication use. During the home visit, the PN registered answers to the items included in the [G]OLD-instrument manually on a structured registration form. For each test or question in the CGA, evidence-based or practice-based cut-off points were provided to help decide whether a problem required further attention (dichotomous).

\subsubsection{Care plan}

The care plan used by PNs originated from the format as recommended by the Dutch College of General Practitioners ${ }^{[12]}$. For each problem, PNs were requested to indicate the goal to be reached; who will undertake what action; and at what date evaluation will take place. A case-manager was assigned who is responsible for care coordination, 
monitoring, and evaluation. PNs received brief training how to use the care plan, as this aspect was covered extensively during their training as PN. A researcher (MS) and project assistant (MM) compared the results of the CGA to the formulated care plan for each case in order to determine whether problems had been included (dichotomous).

\subsubsection{Semi-structured interviews}

The semi-structured interviews $(n=12)$ were digitally recorded and transcribed verbatim. Interviews took place between May and July 2012 with each PN individually at the general practice. Each interview began by asking open-ended questions regarding which factors in general influenced the decision to register detected problems in the care plan. Subsequently, each selected case was discussed with the PN. Questions included whether a problem in the care plan was monitored and evaluated, and reasons per detected problem for not including it in the care plan or not having monitored it during follow-up. Interviews lasted on average 50.2 minutes ( $\mathrm{SD}=10.6$; range: 39.2-68.5).

\subsection{Data analysis}

Qualitative data were analysed using NVivo version 7, in which transcripts were transferred anonymously. A researcher (MS) and project assistant (MM) coded the transcripts independently and parallel. Two sets of categories emerged that were combined after comparing for overlap. Any inconsistencies were resolved through discussion with other members of the research team (HV, ID, and MJ). A general inductive approach ${ }^{[13]}$ was applied for data analysis to derive findings in the context of the research questions guiding this study. Systematic and rigorous reading and coding of the transcripts allowed major themes to emerge.

Parts of the qualitative data (i.e., how the care plan for each case was monitored and evaluated) were converted into numerical codes that were then analysed quantitatively using SPSS version 17.0. Furthermore, the results of the CGA in terms of detected problems and needs (dichotomous) per older person were compared with the accompanying care plan (problem included or not included; dichotomous). Descriptive statistics (e.g., frequencies) were computed for quantitative data.

\section{Results}

\subsection{Characteristics practice nurses}

Participating PNs had a mean age of 40.9 years ( $\mathrm{SD}=10.2$; range: 28.6-59.1) and were predominantly female (91.7\%). PNs had an educational background in nursing (33.3\%), nursing complemented with training as PN (33.3\%), physician assistance complemented with training as PN (25.0\%), and psychology complemented with training as PN (8.4\%). Their mean working experience as $\mathrm{PN}$ was 4.5 years $(\mathrm{SD}=1.8$; range: $2.3-8.2$ ).

\subsection{Characteristics older people}

The age of selected cases at the time of the home visit ranged from 74.8-95.5 years ( $\mathrm{Mdn}=80.6)$ and $54.2 \%$ was female. They did not statistically differ from the remaining 445 older people visited by the PNs with respect to age, $U=24317.50$, $P=.15, r=-0.06$ and gender, $\chi^{2}=0.70, \mathrm{df}=1, P=.40$. As intended, significantly more problems were detected among the selected cases ( $\mathrm{Mdn}=8.0$; range: 6-10) compared to those not included in this study ( $\mathrm{Mdn}=5.0$; range: 3-7), $U=11302.00$, $P<.001, r=-0.41$.

\subsection{Formulation of care plan}

\subsubsection{Actions in general}

For 9 cases (7.5\%), PNs considered a care plan to be unnecessary (Mdn $=7.0$ problems; range: $5-8.5)$ (e.g., detection of known and already addressed problems). For 94 cases (78.3\%), they formulated a care plan according to the format of the 
Dutch College of General Practitioners (Mdn = 8.0 problems; range: 6-10). Two PNs documented follow-up actions in the GP Information System for 17 cases (14.2\%) (Mdn =9.0 problems; range: 7-12). Since these did not contain all necessary details, we did not count them as care plans. The average percentage of detected problems included in care plans (completeness) varied considerably between PNs (range: 10.8\%-52.9\%).

\subsubsection{Actions per problem}

In total, 989 problems were detected among the cases of which $27.8 \%(n=275)$ were included in a care plan. Problems/ needs most often included were need for help in (I) ADL (55.6\%), financial problems (50.0\%), and depressive complaints (45.5\%). Least often included were smoking $(0 \%)$, BMI $<20$ or $>27(1.8 \%)$, and health deterioration in past 12 months (3.5\%) (see Table 1). The reason mentioned most by PNs for not including specific problems in the care plan was that it was an existing problem and/or action had already been undertaken (13.5\%) (see Table 2).

Table 1. Course of action practice nurse per detected problem in the CGA among selected cases $(\mathrm{n}=120)$

\begin{tabular}{|c|c|c|c|c|c|c|c|}
\hline \multirow{2}{*}{$\begin{array}{l}\text { (Early signs of) problems according to } \\
\text { cut-off points comprehensive geriatric assessment }\end{array}$} & \multirow{2}{*}{$N$} & \multicolumn{2}{|c|}{ Prevalence } & \multicolumn{2}{|c|}{ Included in plan } & \multicolumn{2}{|c|}{ Action undertaken } \\
\hline & & $n$ & $\%$ & $n$ & $\%^{*}$ & $n$ & $\%^{\dagger}$ \\
\hline \multicolumn{8}{|l|}{ General health problems } \\
\hline Complaints past $1-2$ months & 120 & 74 & 61.7 & 22 & 29.7 & 14 & 63.6 \\
\hline Health deterioration in past 12 months & 120 & 57 & 47.5 & 2 & 3.5 & 2 & 100 \\
\hline \multicolumn{8}{|l|}{ Problems medication use } \\
\hline Polypharmacy (daily intake of $\geq 5$ medications) & 117 & 67 & 57.3 & 29 & 43.3 & 17 & 58.6 \\
\hline Discrepancy registered and actual medication use & 114 & 17 & 14.9 & 4 & 23.5 & 4 & 100 \\
\hline \multicolumn{8}{|l|}{ Physical problems and lifestyle } \\
\hline Systolic blood pressure $>140 \mathrm{mmHg}$ & 119 & 64 & 53.8 & 29 & 45.3 & 25 & 86.2 \\
\hline Fall incidents in past 6 months & 120 & 62 & 51.7 & 21 & 33.9 & 12 & 57.1 \\
\hline Body Mass Index $(\mathrm{BMI})<20$ or $>27 \mathrm{~kg} / \mathrm{m}^{2}$ & 109 & 56 & 51.4 & 1 & 1.8 & 1 & 100 \\
\hline Insufficient physical activity & 119 & 59 & 49.6 & 6 & 10.2 & 4 & 66.7 \\
\hline Impaired hearing & 110 & 51 & 46.4 & 19 & 37.3 & 10 & 52.6 \\
\hline Urine incontinence & 120 & 53 & 44.2 & 10 & 18.9 & 6 & 60.0 \\
\hline Decreased mobility & 119 & 52 & 43.7 & 17 & 32.7 & 12 & 70.6 \\
\hline Impaired sight & 119 & 51 & 42.9 & 13 & 25.5 & 6 & 46.2 \\
\hline Decreased appetite & 119 & 20 & 16.8 & 1 & 5.0 & 0 & 0 \\
\hline Need for help (instrumental) activities of daily living & 116 & 18 & 15.5 & 10 & 55.6 & 8 & 80.0 \\
\hline Smoking & 119 & 14 & 11.8 & 0 & 0 & 0 & 0 \\
\hline Excessive alcohol use & 76 & 6 & 7.9 & 1 & 16.7 & 0 & 0 \\
\hline Undesired weight loss & 112 & 7 & 6.3 & 2 & 28.6 & 1 & 50.0 \\
\hline Faecal incontinence & 120 & 6 & 5.0 & 1 & 16.7 & 1 & 100 \\
\hline \multicolumn{8}{|l|}{ Psychological and mental problems } \\
\hline Memory problems & 118 & 96 & 81.4 & 39 & 40.6 & 20 & 51.3 \\
\hline Personality disorder & 119 & 30 & 25.2 & 6 & 20.0 & 1 & 16.7 \\
\hline Concerned about falling & 120 & 28 & 23.3 & 9 & 32.1 & 8 & 88.9 \\
\hline Depressive complaints & 120 & 22 & 18.3 & 10 & 45.5 & 5 & 50.0 \\
\hline Anxiety & 120 & 12 & 10.0 & 4 & 33.3 & 2 & 50.0 \\
\hline \multicolumn{8}{|l|}{ Social problems } \\
\hline Burdened by informal care giving & 19 & 8 & 42.1 & 3 & 37.5 & 2 & 66.7 \\
\hline Concerns about informal caregiver & 54 & 14 & 25.9 & 3 & 21.4 & 2 & 66.7 \\
\hline Loneliness & 119 & 25 & 21.0 & 7 & 28.0 & 7 & 100 \\
\hline Absence of social network & 115 & 8 & 7.0 & 1 & 12.5 & 0 & 0 \\
\hline Unsatisfied with daily routines & 119 & 8 & 6.7 & 3 & 37.5 & 2 & 66.7 \\
\hline Financial problems & 119 & 4 & 3.4 & 2 & 50.0 & 1 & 50.0 \\
\hline
\end{tabular}

"Percentages are based on the numbers presented in the column 'Prevalence'.

† Percentages are based on the numbers presented in the column 'Included in plan'. 
Table 2. Reasons practice nurses for not including a specific problem in the care plan ( $n=714$ problems)

\begin{tabular}{lll}
\hline & $\boldsymbol{n}$ & $\%$ \\
\hline Existing problem and/or action has already been undertaken & 96 & 13.5 \\
Older person does not wish any action & 53 & 7.4 \\
Score just below cut-off point, no reason yet for further action & 36 & 5.1 \\
No change possible anymore & 24 & 3.4 \\
Advice given, no further action needed & 21 & 2.9 \\
Not considered a problem (anymore) by the older person & 19 & 2.7 \\
Additional examination revealed it was not a problem after all & 18 & 2.5 \\
Explicable, no further action needed according to the GP & 11 & 1.5 \\
Older person undertakes action himself/herself & 3 & 0.4 \\
Watchful waiting policy & 3 & 0.4 \\
No appropriate services available to address problem & 1 & 0.1 \\
Other: PN did not formulate a care plan & 217 & 30.4 \\
Other: Reason unknown & 212 & 29.7 \\
\hline
\end{tabular}

\subsubsection{Practice nurses' views}

Besides the cut-off points in the [G]OLD-instrument, other factors influenced the decision to include a problem in the care plan, such as the seriousness of the problem:

[...] to what extent does it often return during the conversation, a particular subject. How emotionally charged is it. And well, then I think like if something is a one-time event then I do not immediately consider it a problem. (PN8)

In addition, the older person's wishes were considered:

If the client clearly says I don't want that and I have the feeling like well, I dare to make the decision to not do anything with it, then I also leave it like that. (PN10)

Another factor involved to what extent undertaking action was still attainable at the older person's age:

And then the question is do you still want to undertake such things if people have a certain age, so to say. (PN6)

Finally, the GP's opinion and available knowledge concerning the older person's health status played a role in deciding whether to include a problem in the care plan:

And, we've had this as well, that just ... that someone did not know one word that well or did the clock slightly wrong and that from the experience the GP had with this person, we said like at this moment we don't do anything with it. (PN2)

Lifestyle-related behaviours such as smoking and physical exercise were often not included in the plan, because PNs assumed that dietary and lifestyle patterns were addressed in other disease management programmes, action often only consisted of providing advice, or older people were reluctant to change their behaviour.

\subsection{Adherence to care plan and monitoring during follow-up}

\subsubsection{Actions in general}

Of the 120 cases, $71.7 \%(n=86)$ were monitored and this mainly took place via other disease management programmes $(n$ $=35,29.2 \%$ ) (see Table 3). The PN who performed the home visit was most often responsible for monitoring and evaluation. 
Table 3. Arrangement long-term monitoring of older people by practice nurses after the initial home visit $(\mathrm{n}=120)$

\begin{tabular}{lll}
\hline & $\mathbf{n}$ & $\%$ \\
\hline Via contacts on behalf of disease management programmes & 35 & 29.2 \\
Home visits at fixed time points & 21 & 17.5 \\
PN assigned monitoring to another caregiver (formal or informal) & 19 & 15.8 \\
Mutual agreement that older person contacts PN when needed & 5 & 4.2 \\
Telephone calls at fixed time points & 3 & 2.5 \\
Consultation in the general practice at fixed time points $^{*}$ No monitoring $^{*}$ & 3 & 2.5 \\
\hline
\end{tabular}

* No monitoring because no further actions were needed $(\mathrm{n}=16,47.1 \%)$, older person is lost out of sight $(\mathrm{n}=10,29.4 \%)$, or an event in the older person's life causing discontinuation of monitoring (e.g., illness, death, left the practice, admission to nursing home) $(n=8,23.5 \%)$.

\subsubsection{Actions per problem}

In total, 173 problems were addressed by means of follow-up actions, which is $62.9 \%$ of all problems registered in a care plan $(n=275)$ and $17.5 \%$ of all identified problems through the CGA $(n=989)$. The problems addressed most often were health deterioration in past 12 months, discrepancy between registered and actual medication use, BMI $<20$ or $>27$, faecal incontinence, and loneliness (all 100\%). Decreased appetite, smoking, excessive alcohol use, and absence of social network were dealt with the least (all 0\%) (see Table 1). Most often, actions according to the care plan were not undertaken because the older person did not wish so (36.3\%) (see Table 4).

Table 4. Reasons practice nurses for not undertaking action for problems in the care plan ( $n=102$ problems)

\begin{tabular}{lll}
\hline & $\boldsymbol{n}$ & \% \\
\hline Older person does not wish any action & 37 & 36.3 \\
Existing problem and/or action has already been undertaken & 20 & 19.6 \\
Due to older person's medical circumstances (e.g., ill, admitted to nursing home or home for & 11 & 10.8 \\
older people, deceased) & 9 & 8.8 \\
Additional examination revealed it was not a problem after all & 8 & 7.8 \\
No change possible anymore & 4 & 3.9 \\
Forgotten to monitor & 4 & 3.9 \\
Not considered a problem (anymore) by the older person & 3 & 2.9 \\
Not necessary anymore, older person's status improved & 2 & 2.0 \\
Watchful waiting policy & 2 & 2.0 \\
Explicable, no further action needed according to the GP & 2 & 2.0 \\
PN cannot recall reason & & \\
\hline
\end{tabular}

\subsubsection{Practice nurses' views}

In general, PNs deemed monitoring of older people over time important to get an overview of the success rate of the follow-up actions undertaken. However, sometimes there was no need for monitoring (e.g., when only advice was given) or monitoring was considered unnecessary once the goal according to the care plan was reached. No time for monitoring and evaluation was a prominent issue raised and most PNs struggled how to shape the process of monitoring given the limited hours they could dedicate to care for older people:

So now, I only spend four hours a week to care for older people. [...] And then I notice that sometimes that goes by the board you know, I am honest in that. Then I think, actually I would want to call these people now to see how they are doing, what to do next. (PN2)

PNs often encountered older people who refused follow-up actions (care avoiders) and who only agreed with actions to be undertaken when their health status decreased significantly. These people challenged PNs in how to prevent losing them out of sight:

[...] we decided like make sure to keep in touch, gaining a bit of confidence and then little by little you try to start some care in this way. (PN1) 


\section{Discussion}

Besides case management for people with chronic diseases, practice nurses within general practices in the Netherlands are increasingly held responsible for organising care for older people. The present study examined into more detail how PNs formulate and adhere to a care plan in delivering proactive care to community-dwelling older people.

Although nurse-led chronic disease management has proven to be feasible, acceptable, and sustainable in general practice ${ }^{[14]}$, proactive care for older people poses additional challenges to PNs. Concerning the formulation of care plans, only $27.8 \%$ of the problems identified through CGA were included in a care plan, which is in agreement with our previous work ${ }^{[15]}$. It implies, as confirmed by the findings, that the formulation of care plans by PNs is not only influenced by the cut-off points of the CGA, but also by a complex interplay of patient-related and problem-specific factors. In decision-making, PNs may outweigh the importance of various factors based on their knowledge of the older person ${ }^{[16]}$ and other key aspects known to influence nursing decision-making, such as expertise and intuition ${ }^{[17]}$. Moreover, the post-discussion of each CGA with the GP could have influenced the decision to prioritise problems typically identified under a more medical model (e.g., hypertension) at the expense of more client-centred problems. Further research is needed to support this assertion.

Also consistent with our previous work ${ }^{[15]}$, we found that the main reason for not including the remaining $72.2 \%$ of the problems in the care plan was that the problem was already known and/or action had already been undertaken. Hence, it might be argued that the ability of the [G]OLD-instrument to identify new or unmet needs that require follow-up actions is limited. Alternatively, older people who consented to participate in the trial and were visited by PNs may have been those who frequently visit their GP or they are healthier than their non-participating counterparts, suggesting volunteer bias ${ }^{[18]}$.

Although few problems were registered in a care plan, $62.9 \%$ of these problems were dealt with according to PNs, suggesting a high adherence to the care plan approximately 1 year after it was formulated. This percentage is higher than reported by Piccoliori et al. ${ }^{[19]}$ ( $47 \%$ of the problems were dealt with after one year), and might be explained by the involvement of the PN as case-manager who can attribute more time to care planning and coordination than GPs. However, an often-mentioned reason by PNs for not monitoring during follow-up was a lack of time. At the time of our study, general practices were not separately paid for care coordination and other services performed outside the initial home visit. As a result, PNs may not have been able to devote as much time to care coordination as they wanted. The importance of time in PNs' adherence to case management elements has been identified before ${ }^{[20,21]}$.

The findings imply that training for PNs targeted at acquiring additional knowledge and skills in formulating care plans may improve its adequate formulation and equalise undesired variation between PNs in the completeness of formulated care plans. In addition, the recommended format for the care plan may require adjustments to fit PNs' needs. Furthermore, PNs should be allowed sufficient time to monitor and evaluate care provided to older people. With the increased workload imposed on PNs, these case management steps are most likely to be discarded first. To deal with this issue, a transition from the current fee-for-service payments to a bundled payment system (i.e., health insurers pay a single fee to a multidisciplinary care group to cover different elements of care, for example for patients with chronic diseases) ${ }^{[22]}$ may be considered.

A limitation of the current study is that PNs and the researcher who conducted the interviews knew one another from earlier contacts on behalf of the trial. Although this may have influenced the answers given by PNs, it can also have created a relationship of trust and thereby fostered sincerity in PNs' responses. The researcher's independent role and asking questions in a non-offending way concerning a possibly sensitive subject as adherence to case management steps may also have been conducive to honest responding by PNs. The questions asked necessitated PN to recall their actions regarding care plans they had formulated at least a year ago. Nonetheless, PNs gave detailed answers to the questions in most instances, suggesting a small possibility of recall bias. A second limitation is that during the quantification process of parts of the semi-structured interviews, some numerical codes could not be inferred easily from the interview data due to a lack 
of detailed questioning by the interviewer or insufficient detail in answers given by PNs (see Table 2, 29.7\% of the reasons for not including a problem in the care plan are unknown). The use of an interview guide could not prevent that PNs often gave general case-related reasons instead of problem-specific reasons for not having included a problem in the care plan or for not having monitored the problem over time.

\section{Conclusion}

Despite high adherence of PNs to care plans, only about one quarter of the identified problems were included in a plan. The completeness of care plans varied between PNs and they experienced difficulties in monitoring older people over time. Additional training of PNs, adapting the care plan to fit PN's needs, and allowing PNs to monitor older people over time may improve the formulation of and adherence to care plans. Further research is needed to verify the current findings among a larger sample of PNs involved in proactive care for older people and by using objective record reviews instead of subjective accounts of PNs' actions.

\section{Acknowledgements}

We would like to thank all general practices for their participation in this study and we thank the practice nurses in particular for their cooperation. We are grateful to Marion de Mooij (MM) for her contribution to the analysis of the qualitative data. This study was funded by the Netherlands Organisation for Health Research and Development ('ZonMw', grant number 311070303) within the National Care for the Elderly Programme.

\section{Competing interests}

The authors declare to have no competing interests.

\section{Funding}

This study was funded by the Netherlands Organisation for Health Research and Development ('ZonMw', grant number 311070303) within the National Care for the Elderly Programme.

\section{References}

[1] Fried LP, Ferrucci L, Darer J, Williamson JD, Anderson G. Untangling the concepts of disability, frailty, and comorbidity: implications for improved targeting and care. J Gerontol A Biol Sci Med Sci. 2004; 59: 255-263. http://dx.doi.org/10.1093/gerona/59.3.M255

[2] Lacas A, Rockwood K. Frailty in primary care: a review of its conceptualization and implications for practice. BMC Med. 2012; 10: 4. http://dx.doi.org/10.1186/1741-7015-10-4

[3] Laurant M, Reeves D, Hermens R, Braspenning J, Grol R, Sibbald B. Substitution of doctors by nurses in primary care. Cochrane Database Syst Rev. 2004, 4: Art. No.: CD001271.

[4] Battersby M, Von Korff M, Schaefer J, Davis C, Ludman E, Greene SM, et al. Twelve evidence-based principles for implementing self-management support in primary care. Jt Comm J Qual Patient Saf. 2010; 36: 561-570. PMid:21222358

[5] Stijnen MMN, Duimel-Peeters IGP, Jansen MWJ, Vrijhoef HJM. Early detection of health problems in potentially frail community-dwelling older people by general practices - project [G]OLD: design of a longitudinal, quasi-experimental study. BMC Geriatr 2013;13:7. http://dx.doi.org/10.1186/1471-2318-13-7

[6] Huss A, Stuck AE, Rubenstein LZ, Egger M, Clough-Gorr KM. Multidimensional preventive home visit programs for community-dwelling older adults: a systematic review and meta-analysis of randomized controlled trials. J Gerontol A Biol Sci Med Sci. 2008; 63A: 298-307. http://dx.doi.org/10.1093/gerona/63.3.298

[7] Markle-Reid M, Browne G, Weir R, Gafni A, Roberts J, Henderson SR. The effectiveness and efficiency of home-based nursing health promotion for older people: a review of the literature. Med Care Res Rev. 2006; 63: 531-569. http://dx.doi.org/10.1177/1077558706290941

[8] Stuck AE, Siu AL, Wieland GD, Adams J, Rubenstein LZ. Comprehensive geriatric assessment: a meta-analysis of controlled trials. Lancet. 1993; 342: 1032-1036. http://dx.doi.org/10.1016/0140-6736(93)92884-V 
[9] Reuben DB, Roth C, Kamberg C, Wenger NS. Reconstructing primary care practices to manage geriatric syndromes: the ACOVE-2 intervention. J Am Geriatr Soc. 2003; 51: 1787-1793. http://dx.doi.org/10.1046/j.1532-5415.2003.51565.x

[10] Metzelthin SF, Daniëls R, Van Rossum E, Cox K, Habets H, De Witte LP, et al. A nurse-led interdisciplinary primary care approach to prevent disability among community-dwelling frail older people: a large-scale process evaluation. Int J Nurs Stud. 2013; 50: 1184-1196. http://dx.doi.org/10.016/j.ijnurstu.2012.12.016

[11] Edmonds WA, Kennedy TD. An applied reference guide to research designs. Quantitative, qualitative and mixed methods. Thousand Oaks, CA: Sage; 2013.

[12] Dutch College of General Practitioners: NHG-Praktijkwijzer Ouderenzorg [Dutch College of General Practioners - Manual care for older people in general practice]. Utrecht: NHG; 2010.

[13] Thomas DR. A general inductive approach for analyzing qualitative evaluation data. Am J Eval. 2006; 27: $237-246$. http://dx.doi.org/10.1177/1098214005283748

[14] Hegney DG, Patterson E, Eley DS, Mahomed R, Young J. The feasibility, acceptability and sustainability of nurse-led chronic disease management in Australian general practice: the perspectives of key stakeholders. Int J Nurs Pract. 2013; 19 : 54-59. PMid:23432889 http://dx.doi.org/10.1111/ijn.12027

[15] Stijnen MMN, Jansen MWJ, Vrijhoef HJM, Duimel-Peeters IGP. Development of a home visitation programme for the early detection of health problems in potentially frail community-dwelling older people by general practices. Eur J Ageing 2013;10:49-60. http://dx.doi.org/ 10.1007/s10433-012-0251-7

[16] Funkesson KH, Anbäcken EM, Ek AC. Nurses' reasoning process during care planning taking pressure ulcer prevention as an example. A think-aloud study. Int J Nurs Stud. 2007; 44: 1109-1119. http://dx.doi.org/10.1177/1098214005283748

[17] Lamb B, Sevdalis N. How do nurses make decisions? Int J Nurs Stud. 2011; 48: 281-284. http://dx.doi.org/10.1016/j.ijnurstu.2010.10.003

[18] Van Heuvelen MJG, Hochstenbach JBM, Brouwer WH, De Greef MHG, Zijlstra GAR, Jaarsveld E, et al. Differences between participants and non-participants in an RCT on physical activity and psychological interventions for older persons. Aging Clin Exp Res. 2005; 17: 236-245.

[19] Piccoliori G, Gerolimon E, Abholz H. Geriatric assessment in general practice using a screening instrument: is it worth the effort? Results of a South Tyrol Study. Age Ageing. 2008; 37: 647-652. http://dx.doi.org/10.1093/ageing/afn161

[20] Evans C, Drennan V, Roberts J. Practice nurses and older people: a case management approach to care. J Adv Nurs. 2005; 51: 343-352. http://dx.doi.org/10.1111/j.1365-2648.2005.03504.x

[21] Boase S, Kim Y, Craven A, Cohn S. Involving practice nurses in primary care research: the experience of multiple and competing demands. J Adv Nurs. 2011; 68: 590-599. http://dx.doi.org/10.1111/j.1365-2648.2011.05764.x

[22] De Bakker DH, Struijs JN, Baan CA, Raams J, De Wildt J, Vrijhoef HJM, et al. Early results from adoption of bundled payment for diabetes care in the Netherlands show improvement in care coordination. Health Aff (Millwood). 2012; 31: $426-433$. http://dx.doi.org/10.1377/hlthaff.2011.0912 